\title{
POLYNOMIAL APPROXIMATION FOR A CLASS OF PHYSICAL RANDOM VARIABLES
}

\author{
A. DE SANTIS, A. GANDOLFI, A. GERMANI, AND P. TARDELLI
}

(Communicated by James Glimm)

\begin{abstract}
In white noise theory on Hilbert spaces, it is known that maps which are uniformly continuous around the origin in the S-topology constitute an important class of "physical" random variables. We prove that random variables having such a continuity property can be approximated in the gaussian measure by polynomial random variables. The proof relies on representing functions which are uniformly S-continuous around the origin as the composition of a continuous map with a Hilbert-Schmidt operator.
\end{abstract}

\section{INTRODUCTION}

Weak distribution theory and its associate white noise theory have provided, in recent years, an alternative approach to the usual Itô calculus for modeling physical systems in a noisy environment $[1,2]$. The main concepts of this approach, which from the point of view of engineering application seems to be more useful than the conventional one, can briefly be outlined as follows.

Any physical signal taking values in a separable Hilbert space $H$ and observed in a finite time interval $[0, T]$ has bounded variation and finite energy. Thus a natural space to model it is $\mathscr{H}=L_{2}([0, T] ; H)$. Gaussian noise has a bandwidth much larger than the signal one. It follows that the white noise can be well modelled as the identity map on $\mathscr{H}$ endowed with the gaussian measure $\mu_{\mathrm{G}}$ with identity covariance operator. The measure $\mu_{\mathrm{G}}$ has the following characteristic functional:

$$
C_{\mu_{\mathrm{G}}}(h)=\int_{\mathscr{R}} e^{i[h, \omega]} d \mu_{\mathrm{G}}(\omega)=e^{\|h\|^{2} / 2} .
$$

Then $\mu_{\mathrm{G}}$ is defined only on the algebra $\mathscr{C}$ of cylinder sets of $\mathscr{H}$ and cannot be extended to the whole Borel $\sigma$-algebra $\mathscr{B}_{\mathscr{C}}$ on $\mathscr{H}$ [3].

Let a physical device be described by the measurable map $F: \mathscr{H} \rightarrow \mathscr{H}^{\prime}, \mathscr{H}$ and $\mathscr{H}^{\prime}$ real separable Hilbert spaces. It is of primary importance to be able to compute

$$
\mu_{\mathrm{G}}(\omega: F(\omega) \in B), \quad B \in \mathscr{B}_{\mathscr{H}^{\prime}},
$$

Received by the editors April 25, 1992.

1991 Mathematics Subject Classification. Primary 60B11, 47H99, 46N30.

This work was partially supported (40\%) by grant MPI. 
where $\mathscr{B}_{\mathscr{H}}$, is the Borel $\sigma$-algebra on $\mathscr{H}^{\prime}$. This computation in general is not possible because $\{\omega: F(\omega) \in B\}$ in general does not belong to $\mathscr{C}$. On the other hand the family of sets

$$
\mathscr{F}^{F}=\left\{F^{-1}(B), B \in \mathscr{B}_{\mathscr{H}^{\prime}}\right\}
$$

is a sub- $\sigma$-algebra of $\mathscr{B}_{\mathscr{H}}$ so that, for some measurable $F, \mu_{\mathrm{G}}$ can be extended to $\mathscr{F} F$. In this case we can qualify $F$ to be a $\mu_{\mathrm{G}}$-random variable (R.V.) and $\mu_{\mathrm{G}} \circ F^{-1}$ is countably additive on $\left(\mathscr{H}^{\prime}, \mathscr{B}_{\mathscr{H}^{\prime}}\right)$.

Any measurable function of type $F \circ P_{n}, P_{n}$ being a finite-dimensional projector, is obviously a $\mu_{G}-R$.V. Such a class of R.V.s is rather poor for applications; an important larger class of $\mu_{\mathrm{G}}-\mathrm{R}$.V. is the class of the so-called physical random variables (P.R.V.) introduced by Balakrishnan [3].

Let $\mathscr{P}(\mathscr{H})$ denote the set of all sequences of finite-dimensional projectors on $\mathscr{H}$ increasing strongly to the identity. We recall the following definition of a physical random variable $[3,4]$.

Definition 1. Let $\left(\mathscr{H}, \mathscr{C}, \mu_{\mathrm{G}}\right)$ be a cylinder-probability triple on the real separable Hilvert space $\mathscr{H}$ endowed with the finite additive cylinder measure $\mu_{\mathrm{G}}$. Then the function $F:\left(\mathscr{H}, \mathscr{C}, \mu_{\mathrm{G}}\right) \rightarrow \mathscr{B}, \mathscr{B}$ a real separable Banach space, is a physical random variable (P.R.V.) if

(i) $\left\{F \circ P_{n}\right\}$ is Cauchy in $\mu_{\mathrm{G}}$-measure for every sequence $\left\{P_{n}\right\} \in \mathscr{P}(\mathscr{H})$, and

(ii) the sequence $\left\{\nu_{n}\right\}$ of probability measures on $\mathscr{B}$ defined by $\nu_{n}=\mu_{\mathrm{G}}$ 。 $\left(F \circ P_{n}\right)^{-1}$ converges strongly to the same probability measure $\nu$ on $\mathscr{B}$ for every sequence $\left\{P_{n}\right\} \in \mathscr{P}(\mathscr{H})$.

When $\mathscr{B}$ is a Hilbert space, condition (ii) is equivalent to saying that the limit

$$
C(h)=\lim _{n \rightarrow \infty} \int_{\mathscr{H}} e^{i\left[F \circ P_{n} \omega, h\right]} d \mu_{\mathrm{G}}(\omega)
$$

is independent of the sequence $\left\{P_{n}\right\}$ chosen.

In the P.R.V. class we can always extend $\mu_{\mathrm{G}}$ to events in $\mathscr{F} F$ by

$$
\mu_{\mathrm{G}} \circ F^{-1}(B)=\lim _{n \rightarrow \infty} \mu_{\mathrm{G}}\left(\omega \in \mathscr{H}: F \circ P_{n} \omega \in B\right), \quad B \in \mathscr{B}_{\mathscr{B}} .
$$

The class of all $\mathscr{B}$-valued P.R.V.'s is denoted by $\mathscr{L}^{\prime}\left(\mathscr{H}, \mathscr{C}, \mu_{\mathrm{G}} ; \mathscr{B}\right)$. We need to introduce the following continuity concepts in order to characterize an important subclass of $\mathscr{L}^{\prime}\left(\mathscr{H}, \mathscr{C}, \mu_{\mathrm{G}} ; \mathscr{B}\right)$.

Definition 2. A map $F: \mathscr{H} \rightarrow \mathscr{B}$ is continuous in $x \in \mathscr{H}$ with respect to the S-topology (Sazonov topology) if, for any $\varepsilon>0$, there exists an H.S. operator $L_{\varepsilon}(x): \mathscr{H} \rightarrow \mathscr{H}$ such that

$$
\left\|L_{\varepsilon}(x)\left(x-x^{\prime}\right)\right\|<1
$$

implies

$$
\left\|F(x)-F\left(x^{\prime}\right)\right\|<\varepsilon .
$$

$F$ is uniformly S-continuous on $U \subset \mathscr{H}$ when the H.S. operator in (1) does not depend on $x \in U$. 
Definition 3. A map $F: \mathscr{H} \rightarrow \mathscr{B}$ is said to be uniformly S-continuous around the origin (U.S.C.A.O.) if $F$ is uniformly S-continuous on the sets

$$
U_{n}=\left\{x \in \mathscr{H}:\left\|A_{n} x\right\| \leq 1\right\}
$$

where $\left\{A_{n}\right\}_{1}^{\infty}$ is a sequence of H.S. operators such that

$$
\left\|A_{n}\right\|_{\text {H.S. }} \rightarrow 0 \text { and } \bigcup_{n=1}^{\infty} U_{n}=\mathscr{H} \text {. }
$$

Obviously a map which is uniformly S-continuous on $\mathscr{H}$ is also uniformly S-continuous around the origin. A necessary and sufficient condition for a function $F$ to be U.S.C.A.O. is the existence of an H.S. operator $L$ such that $F$ is uniformly S-continuous on the set $U_{m}=\{x \in \mathscr{H}:\|L x\| \leq m\}$ for each integer $m>0$ (see [5, p. 109]). Moreover, the U.S.C.A.O. class is closed with respect to left concatenation with continuous maps $[6,2]$.

The importance of definitions (2) and (3) is justified by the following theorem, whose proof is essentially given in $[6,7]$ (see [8] for a simpler proof).

Theorem 1.1. Let $F:\left(\mathscr{H}, \mathscr{C}, \mu_{\mathrm{G}}\right) \rightarrow \mathscr{B}$ be a U.S.C.A.O. map. Then $F$ is a P.R.V.

When $\mathscr{B}$ is a Hilbert space, note that if $F$ is an H.S. operator then $F$ obviously defines a linear P.R.V.

\section{THE APPROXIMATION THEOREM}

Let $\mathscr{H}$ and $\mathscr{B}$ be a real separable Hilbert space and a real separable Banach space respectively. Let us recall that an $n$-linear operator $M$ on $\mathscr{H} \times \cdots \times \mathscr{H}$ ( $n$ times) into $\mathscr{B}$ is a map which is linear in each of its arguments separately. It is natural to define

$$
M_{n} x^{n}=M(x, \ldots, x)
$$

as the $n$-degree monomial operator (associated to the multilinear operator $M$ ) on $\mathscr{H}$ into $\mathscr{B}$. Then the operator $P: \mathscr{H} \rightarrow \mathscr{B}$, defined as

$$
P(x)=M_{n} x^{n}+M_{n-1} x^{n-1}+\cdots+M_{1} x+M_{0},
$$

where $M_{0}$ stands for a constant operator, is called an $n$-degree polynomial operator. The following generalization of the classical Weierstrass theorem holds $[10,11]$ :

Theorem 2.1. The family of continuous polynomials on $\mathscr{H}$ into $\mathscr{B}$, restricted to a compact set $K$ of $\mathscr{H}$, is dense in the set of continuous functions on $\mathscr{H}$ into $\mathscr{B}$ restricted to $K$.

We will prove that the physical random variables belonging to the U.S.C.A.O. class can be approximated by polynomials. In order to get this result we need the following repiesentation theorem (first appeared in [12]):

Theorem 2.2. The map $F: \mathscr{H} \rightarrow \mathscr{B}$ is uniformly $S$-continuous around the origin if and only if there exists an H.S. operator $\Lambda: \mathscr{H} \rightarrow \mathscr{H}$ and a continuous map $g: \mathscr{H} \rightarrow \mathscr{B}$ such that $F=g \circ \Lambda$.

Proof. In $\S 1$ we saw that a map that is left concatenation of a continuous map with an H.S. operator is in the U.S.C.A.O. class. Only the necessity needs a 
proof. Without loss of generality, let us define the set

$$
U_{m}=\{x \in \mathscr{H}:\|\tilde{\Lambda} x\| \leq m\}, \quad m=1,2, \ldots,
$$

where $\tilde{\Lambda}$ is a suitable H.S. operator such that $F$ is uniformly S-continuous on $U_{m}$ as established in [5]. Therefore, given any $x, y$ in $U_{m}$, it follows that

$$
\|F(x)-F(y)\|<\frac{1}{n}
$$

if, for a suitable H.S. operator $L_{n, m}$, we have

$$
\left\|L_{n, m}(x-y)\right\|^{2}<1,
$$

or equivalently if

$$
\left[\frac{1}{2^{n+m+1}} \frac{L_{n, m}^{*} L_{n, m}}{\left\|L_{n, m}\right\|_{\mathrm{H} . S .}^{2}}(x-y), x-y\right]<\frac{1}{2^{n+m+1}\left\|L_{n, m}\right\|_{\mathrm{H} . \mathrm{S} .}^{2}}=\delta_{n, m} .
$$

Let us introduce the linear operator $R: \mathscr{H} \rightarrow \mathscr{H}$

$$
R=\sum_{n, m} \frac{1}{2^{n+m+1}}\left(\frac{L_{n, m}^{*} L_{n, m}}{\left\|L_{n, m}\right\|_{\text {H.S. }}^{2}}+\frac{1}{n} \pi_{\mathscr{H}}^{n}\right)
$$

where $\left\{\pi_{\mathscr{H}}^{n}\right\}$ is a complete sequence of finite-dimensional orthoprojectors such that $\operatorname{rank}\left(\pi_{\mathscr{H}}^{n}\right)=n$, converging strongly to the identity. The operator $R$ is positive definite, selfadjoint, and trace-class since, denoting by $\left\{e_{i}\right\}$ a complete orthonormal system for $\left\{\pi_{\mathscr{H}}^{n}\right\}$, we have

$$
\begin{aligned}
\sum_{i=1}^{\infty}\left[R e_{i}, e_{i}\right] & =\sum_{i=1}^{\infty}\left[\sum_{n, m} \frac{1}{2^{m+n+1}}\left(\frac{1}{\left\|L_{n, m}\right\|_{\mathrm{H} . \mathrm{S} .}^{2}} L_{n, m}^{*} L_{n, m}+\frac{1}{n} \pi_{\mathscr{H}}^{n}\right) e_{i}, e_{i}\right] \\
& =\sum_{n, m} \frac{1}{2^{n+m+1}} \sum_{i=1}^{\infty}\left(\frac{1}{\left\|L_{n, m}\right\|_{\mathrm{H} . \mathrm{S} .}^{2}}\left[L_{n, m} e_{i}, L_{n, m} e_{i}\right]+\frac{1}{n}\left[\pi_{\mathscr{H}}^{n} e_{i}, e_{i}\right]\right) \\
& =1 .
\end{aligned}
$$

Inequality (3), in view of (5), is also implied by

$$
[R(x-y), x-y]<\delta_{n, m},
$$

since, as can readily be recognized, it is

$$
\left[\frac{1}{2^{n+m+1}} \frac{L_{n, m}^{*} L_{n, m}}{\left\|L_{n, m}\right\|_{\text {H.S. }}^{2}}(x-y), x-y\right] \leq[R(x-y), x-y] .
$$

Now consider the factorization

$$
\Lambda^{*} \Lambda=R+\tilde{\Lambda}^{*} \tilde{\Lambda}
$$

where $\Lambda$ is an H.S. full-rank operator, as can easily be verified by noting that at least $\left\{\left\|\frac{1}{n} \pi_{\mathscr{P}}^{n} x\right\|\right\}$ is a separable sequence of seminorms.

The inverse operator $\Lambda^{-1}$ is well defined, and its domain $\mathscr{D}\left(\Lambda^{-1}\right)$ is dense in $\mathscr{H}$ because of the full-rank property of $\Lambda$. Moreover, let us introduce the sequence of sets

$$
\bar{U}_{m}=\{x:\|\Lambda x\| \leq m\} .
$$

By taking into account (7) and the positive definiteness of $R$, we have

$$
\bar{U}_{m} \subset U_{m} \text {. }
$$


Let us consider the map $\hat{g}: \mathscr{D}\left(\Lambda^{-1}\right) \rightarrow \mathscr{B}$ defined as $\hat{g}(x)=F \circ \Lambda^{-1} x$. The map $\hat{g}$ is uniformly continuous on the sets $G_{m}$ defined as

$$
G_{m}=\left\{x \in \mathscr{D}\left(\Lambda^{-1}\right),\|x\| \leq m\right\} .
$$

Note first that if $x \in G_{m}$ then $x=\Lambda^{-1} x$ and $\left\|\Lambda\left(\Lambda^{-1} x\right)\right\| \leq m$ so that $\Lambda^{-1} x \in \bar{U}_{m} \subset U_{m}$. Recalling that (6) implies (3) in $U_{m}$, for any $x, y \in G_{m}$, it is

$$
\|\hat{g}(x)-\hat{g}(y)\|=\left\|F \circ \Lambda^{-1} x-F \circ \Lambda^{-1} y\right\|<1 / n
$$

if

$$
\left[R\left(\Lambda^{-1} x-\Lambda^{-1} y\right), \Lambda^{-1} x-\Lambda^{-1} y\right]<\delta_{n, m} .
$$

From (7) it is $\Lambda^{*} \Lambda \geq R$, so that (8) is implied by

$$
\left\|\Lambda\left(\Lambda^{-1} x-\Lambda^{-1} y\right)\right\|^{2}=\|x-y\|^{2}<\delta_{n, m}
$$

which proves the uniform continuity of $\hat{g}$ on $G_{m}$. Finally, denoting by $g: \mathscr{H}$ $\rightarrow \mathscr{B}$ the unique continuous extension of $\hat{g}$ on the whole Hilbert space $\mathscr{H}$, it results that $F=g \circ \Lambda$, which proves the theorem.

Remark. Note that the function $g$ is uniformly continuous on any closed sphere. There is no contradiction with the sufficient part of the theorem in that any H.S. operator $L$ can be decomposed in the product of a compact operator $C$ with an H.S. operator $\Lambda$ [7, Lemma 3.6.1]. Thus, if $f$ is a continuous map, $f \circ L=f \circ C \circ \Lambda$, and the map $g=f \circ C$ is clearly uniformly continuous on the closed spheres.

Now we can state the following approximation theorem.

Theorem 2.3. Let $F:\left(\mathscr{H}, \mathscr{C}, \mu_{\mathrm{G}}\right) \rightarrow \mathscr{B}$ be a P.R.V. belonging to the U.S.C.A.O. class. Then, for each $\varepsilon>0$, there exists a polynomial P.R.V. $P_{\varepsilon}$ such that

$$
\mu_{\mathrm{G}}\left(x \in \mathscr{H}:\left\|P_{\varepsilon}(x)-F(x)\right\|>\varepsilon\right)<\varepsilon .
$$

Proof. By Theorem 2.2 there exists an H.S. operator $\Lambda$ and a continuous function $g$ such that $F=g \circ \Lambda$. Given $\varepsilon>0$, by a well-known Prohorov theorem [9] there exists a compact set $C_{\varepsilon}$ in $\mathscr{H}$ such that

$$
\mu_{\mathrm{G}} \circ \Lambda^{-1}\left(C_{\varepsilon}\right)>1-\varepsilon,
$$

since $\mu_{\mathrm{G}} \circ \Lambda^{-1}$ is obviously countably additive. Moreover, by the Weierstrass Theorem 2.1, there exists an $n_{\varepsilon}$-degree polynomial operator $p^{\left(n_{\varepsilon}\right)}$ such that on the compact set $C_{\varepsilon}$

$$
\sup _{x \in C_{\varepsilon}}\left\|p^{\left(n_{\varepsilon}\right)}(x)-g(x)\right\| \leq \varepsilon / 2 \text {. }
$$

The map $p^{\left(n_{\varepsilon}\right)} \circ \Lambda$, being the composition of a continuous map with an H.S. operator, is a P.R.V. by Theorems 1.1 and 2.2 , and obviously it is a polynomial too. We have

$$
\begin{aligned}
& \mu_{\mathrm{G}}\left(\omega:\left\|p^{\left(n_{\varepsilon}\right)}(\Lambda \omega)-g \circ \Lambda \omega\right\|>\varepsilon\right) \\
&=\mu_{\mathrm{G}}\left(\left\{\omega:\left\|p^{\left(n_{\varepsilon}\right)}(\Lambda \omega)-g \circ \Lambda \omega\right\|>\varepsilon, \Lambda \omega \in C_{\varepsilon}\right\}\right. \\
&\left.\quad \cup\left\{\omega:\left\|p^{\left(n_{\varepsilon}\right)}(\Lambda \omega)-g \circ \Lambda \omega\right\|>\varepsilon, \Lambda \omega \notin C_{\varepsilon}\right\}\right) \\
& \leq \mu_{\mathrm{G}}\left(\omega: \Lambda \omega \notin C_{\varepsilon}\right)<\varepsilon,
\end{aligned}
$$

so that the P.R.V. $P_{\varepsilon}=p^{\left(n_{\varepsilon}\right)} \circ \Lambda$ approximates $F$ in the $\mu_{\mathrm{G}}$-measure. 


\section{CONCLUdING REMARKS}

Theorem 2.2 allows us to recognize that the property of being a physical random variable owned by nonlinear transformations uniformly S-continuous around the origin depends essentially on the Hilbert-Schmidt character of a linear transformation. The decomposition stated by this theorem allows, moreover, a simple characterization of an important ciass of P.R.V.s as polynomial maps. These results can be a useful tool in modeling stochastic systems and in filtering applications [13]. We recall here that the P.R.V. class considered in Theorem 2.3 contains the Radon-Nikodým derivative with respect to the gaussian measure of the measure generated by the output of a stochastic system [12].

\section{REFERENCES}

1. A. V. Balakrishnan, Parameter estimation in stochastic differential systems: theory and application, Developments in Statistics, vol. 1, Academic Press, New York, 1978.

2. G. Kallianpur and R. Karandikar, White noise calculus and non-linear filtering theory, Ann. Probab. 13 (1985), 1033-1107.

3. A. V. Balakrishnan, Applied functional analysis, Springer, New York, 1981.

4. A. Germani and P. Sen, White noise solution for a class of distributed feedback systems with multiplicative noise, Ricerche Automat. 10 (1979), 38-65.

5. H. H. Kuo, Gaussian measures in Banach Spaces, Lecture Notes in Math., vol. 463, Springer, New York, 1975.

6. L. Gross, Integration and non-linear transformations in Hilbert space, Trans. Amer. Math. Soc. 94 (1960), 404-440.

7. _ Harmonic analysis on Hilbert spaces, Mem. Amer. Math. Soc., no. 46, Amer. Math. Soc., Providence, RI, 1963, pp. 1-62.

8. A. Gandolfi and A. Germani, On the definition of a topology in Hilbert spaces with applications to the White Noise Theory, J. Franklin Inst. 316 (1983), 435-444.

9. K. R. Parthasarathy, Probability measures on metric spaces, Academic Press, New York, 1967.

10. P. M. Prenter, A Weierstrass theorem for normed linear spaces, Bull. Amer. Math. Soc. 75 (1969), 860-862.

11. On polynomial operators and equations, Nonlinear Functional Analysis and Applications, Academic Press, New York, 1971.

12. A. DeSantis, A. Gandolf, A. Germani, and P. Tardelli, A representation theorem for Radon-Nikodym derivatives in the white-noise theory, Proc. Internat. Conf. on Mathematical Theory of Control (Bombay, India, December 10-15, 1990), Marcel Dekker, New York, 1993, pp. 109-123.

13. R. R. Mazumdar and A. Bagchi, A representation result for nonlinear filters, Proc. COMCON 3 (Victoria, Canada, October 15-18, 1991), Vol. 2, UNLV Publications, Las Vegas, 1992, pp. 794-805.

(A. DeSantis and A. Gandolfi) Istituto di ANalisi dei Sistemi ed INformatica del CNR, V. le Manzoni 30, 00185 Roma, Italy

(A. Germani) Dipartimento di Ingegneria Elettrica, Università dell'Aquila, 67100 Monteluco, L'Aquila, ITALY

(P. Tardelli) Dipartimento di Matematica G. Castelnuovo, Università di Roma "La SAPIENZA", P. LE A. Moro 5, 00184 Roma, ItAly 